Results There was no difference between the two groups in clinical characteristic, while the interval time of onset to reperfusion in LVA group was longer and the incidence of Killip 3 grade was higher than those in non-LVA group ( $<<0.05$, respectively). The peak value of plasma IL-8 in LVA was significant higher and the peak time of plasma cTnI was much earlier than those in the non-LVA group $(p<0.05$, respectively). The peak values of plasma IL-8 in LVA group with LVEDP $\geq 18 \mathrm{~mm} \mathrm{Hg}$ were significant higher than that in nonLVA with LVEDP. The peak value of plasma IL-8 in LVA group with LVEDP $<18 \mathrm{~mm} \mathrm{Hg}$ were significant higher than that in non-LVA with LVEDP $(p<0.05)$. At 6th month post-AMI, the value of LVEF, LVESVI, LVEDVI, WMS and LVEDP in non-LVA group were much better than those in LVA group. The values of LVEF, LVESVI, LVEDVI, WMS and LVEDP in non-LVA group at 6th month postAMI were significantly improved as compared with those at the first time after PCI but the values of LVEDVI, WMS, LVEDP were improved in the LVA group. Within the 6th month follow-up, the incidences of angina post-AMI, heart failure of NYHA $\geq 3$ grade and mortality in LVA group were significantly higher than those in the non-LVA group ( $p<0.05$, respectively).

Conclusions The value of plasma IL-8 is significantly increased and correlated closely with left ventricular remodelling status and haemodynamic change in patients with LVA after AMI. It is indicated that the over activity of immune inflammatory medium IL-8 involves in the process of LVA formation and has an important clinic significance in early diagnosis and appreciation of LVA post AMI.

\section{e0653 LEVEL CHANGE OF PLASMA BNP AND THE RELATIONSHIPS TO THE LEFT VENTRICULAR ANEURYSM FORMATION AND CARDIAC PERFORMANCE IN PATIENTS WITH ACUTE MYOCARDIAL INFARCTION}

doi:10.1136/hrt.2010.208967.653

Fu Xianghua, Liu Jun, Gu Xinshun, Wei Yongyun, Fan Weize, Wang Yanbo. The 2nd Hospital of Hebei Medical University, Shijiazhuang, Hebei, China

Objective To investigate the change of brain natriuretic peptide (BNP) in plasma and the relationships with the left ventricular aneurysm (LVA) and cardiac performance in acute myocardial infarction (AMI) patients using left ventriculography (LVG).

Methods A total of 64 patients with primary anterior AMI accompanied LVA diagnosed by LVG were enrolled in this study and divided into LVA group (33 patients) and non-LVA group (31 patients). Plasma BNP was measured. At the immediately after PCI and 6th month after AMI, the parameters of LVEDVI, LVESVI, LVEF, WMS and LVEDP were measured by LVG. The main adverse cardiac events (MACE) were recorded during 24 week after PCI.

Results The peak value of plasma BNP in LVA group was higher and the arrived time of peak values of peak time of plasma BNP was earlier than those in the non-LVA group ( $p<0.01$, respectively). In 5 th day and 24th week after AMI, the values of BNP in LVA group were higher as compared to those in non-LVA group $(p<0.05$, respectively). The peak value of plasma BNP in LVA group, regardless of whether LVEDP $\geq 18 \mathrm{~mm} \mathrm{Hg}$ or $\operatorname{LVEDP}<18 \mathrm{~mm} \mathrm{Hg}$, were higher than that in non-LVA group under the same level of LVEDP $(p<0.05)$. At 6 th month after AMI, the parameters of LVEF, LVESVI, LVEDVI, WMS and LVEDP in non-LVA group were much better as compared to those in LVA group. The peak value of plasma BNP was significantly correlated with LVESVI, LVEDVI, WMS, LVEDP in LVA group ( $p<0.01$, respectively), while negatively correlated with LVEF at primary PCI $(r=-0.72, p<0.01)$. During the 6 months follow-up, the incidence of MACE in LVA group were higher than that in the non-LVA group $(p<0.05)$. The peak value of plasma BNP in LVA group was significantly correlated with the incidence of MACE $(\mathrm{r}=0.56, \mathrm{p}<0.05)$.
Conclusions The value of plasma BNP in the AMI patients with LVA was higher than that with non-LVA, and significantly correlated with left ventricular remodelling status and homodynamic change. It is indicated that the over secretion of BNP is involved in the left ventricular remodelling and the process of LVA formation.

\section{e0654 THE EFFECT OF PREVENTIVE INTRACORONARY ADMINISTRATION OF ANISODAMINE ON MICROCIRCULATION PERFUSION IN MINISWINES WITH AMI}

doi:10.1136/hrt.2010.208967.654

Fu Xianghua, Gu Xinshun, Fan Weize, Wei Yongyun, Hao Guozhen, Jiang Yunfa, Liu Jun. The 2nd Hospital of Hebei Medical University, Shijiazhuang, Hebei, China

Objective Anisodamine is a M-cholinergic receptor inhibitor that plays improvement effectiveness on the microcirculative perfusion. Our previous study had ever shown reversing effect on no-reflow phenomenon (NRP) in the miniswine models with AMI. The purpose of this study was continually to explore the effect of preventive intracoronary administration of anisodamine on myocardial microcirculation in York swines models with AMI by TIMI frame count, TIMI myocardial perfusion grade and haemodynamic parameters.

Methods 18 York swines ( $25 \sim 35 \mathrm{~kg}, 3 \sim 4$ months old) were divided into saline group $(n=9)$ and Anisodamine group $(n=9)$. Immediately after $2 \mathrm{ml}$ saline and $2 \mathrm{mg}$ anisodamine were injected into LAD in the two groups respectively, PMBS were injected into the coronary artery by three times with $5 \mathrm{~min}$ interval, then incidence of NRP was recognised by TIMI frame count and TIMI myocardial perfusion grade. TnI and CK-MB were measured before PMBS injection and at 5, 60, 120, $180 \mathrm{~min}$ after PMBS injection. MPP and PCWP were measured by Swan-Ganz catheter. ECG was recorded before and immediately after PMBS injection and $\Sigma$ ST were calculated. The changes of PR, ORS and OT duration were also compared.

Results 16 of 18 York swines survived in the whole procedure. NRP was found in all of the eight swines in saline group while four of eight swines in anisodamine group. MPP (mean perfusion pressure) was slightly increased by $4.76 \%$ only in anisodamine group ( $p>0.05$ ), while MPP were significant decreased by $25.23 \%, 23.63 \%$, $19.66 \%$ immediately, $3 \mathrm{~min}$ and $1 \mathrm{~h}$ after NRP in saline group $(118.5 \pm 16.2$ vs $88.6 \pm 12.3,118.5 \pm 16.2$ vs $90.5 \pm 14.3,118.5 \pm 16.2$ vs. $95.2 \pm 16.3 \mathrm{~mm} \mathrm{Hg}, \mathrm{p}<0.05)$, respectively, and PCWP, values of $\Sigma S T$ and mycardial Infarct size in anisodamine group were much lower than that in saline group significantly $(p<0.05)$.

Conclusion Preventive intracoronary administration of anisodamine could improve AMI survival rate with NPR by maintaining effective myocardial microcirculation status and raising coronary perfusion pressure as well decreasing the size of myocardial infarction.

\section{e0655 THE EFFECT OF INTRAVENOUS ADMINISTRATION OF TIROFIBAN ON MYOCARDIAL REFLOW AND LEFT VENTRICULAR REMODELLING DURING PCI IN PATIENTS WITH ACUTE MYOCARDIAL INFARCTION}

doi:10.1136/hrt.2010.208967.655

Fu Xianghua, Fan Weize, Jiang Yunfa, Hao Guozhen, Wang Xuechao, Wang Yanbo. The 2nd Hospital of Hebei Medical University, Shijiazhuang, Hebei, China

Objective To investigate the efficacy and safety of platelet glycoprotein IIb/IIIa inhibition (tirofiban) during PCI in AMI patients performed primary PCI.

Methods A total of 96 patients with AMI were randomised to divide into two groups: the tirofiban group (TG, $n=44$,) and the control 
group (CG, $\mathrm{n}=52$ ). Tirofiban was only administrated in the tirofiban group. Before the CAG, enough clopidogrel, aspirin and heparin be used in both groups. The MACE and the haemorrhage events were collected in each group during in-hospital. The lesion and reperfusion of the IRA and myocardial were analyses by QCA and TMPG. The platelet aggregation rate were recorded All patients received UCG 1 week and 24 weeks after PCI to evaluate the heart function. Results There was no significant differences in age, gender, risk factors, pre-angina, the location of the AMI, heart function, and the mean interval from onset to PCI between the two groups. A greater percentage of TIMI 1 flow of IRA was achieved in TG compared with the control group before PCI $(p<0.05)$. The percentage of TIMI 3 flow of IRA after the guild wire first crossing was higher $(p<0.05)$ in TG. The percentage of TIMI 3 flow in TG after PCI was higher than that in CG $(p<0.05)$. The CTFC and slow-reflow phenomenon was fewer $(p<0.05)$ in TG after PCI. The percentage of TMPG beyond 2 grade was higher in TG $p<0.05)$. The value of LVEF 1 week after PCI in TG was higher than that in CG $(p<0.01)$. The platelet aggregation rate in TG was lower after tirofiban administration for $0.25,0.5,2,6$ and $12 \mathrm{~h}$. There was no significant difference in haemorrhage events between the two groups. There was a lower incidence of MACE in TG compared with that in CG during in-hospital and follow up.

Conclusion Intravenous administration of trofiban can inhibit the platelet aggregation, improve the coronary flow of IRA, decrease the incidence of NRP in AMI patients performed PCI, which in turn will improve the heart function and decrease the incidence of MACE. Tirofiban can make more IRA patent before PCI, but do not increase the haemorrhage events.

\section{e0656 THE EFFECT OF RECOMBINANT HUMAN B-TYPE NATRIURETIC PEPTID ON CORONARY CIRCULATION AND RENAL HAEMODYNAMICS IN YORK PIGS MODEL OF ACUTE MYOCARDIAL INFARCTION WITH HEART FAILURE}

doi:10.1136/hrt.2010.208967.656

Fu Xianghua, Zhang Jing, Wang Xuechao, Wang Yanbo, Xue Ling, Fan Weize, Wu Weili, Jiang Yunfa. The 2nd Hospital of Hebei Medical University, Shijiazhuang, Hebei, China

Objective To evaluate the impact of intravenous administration of rhBNP on coronary and renal artery haemodynamics in York pigs model of AMI-ADHF.

Methods 14 York pigs were included in this study. After the AMI$\mathrm{ADHF}$ models were established, pigs were randmized into saline group and rhBNP group. Coronary pressure $\left(\mathrm{P}_{\mathrm{c}}\right)$, the average peak velocity (APV), coronary vascular resistance (CR), coronary flow reserve (CFR) and coronary diameter were recorded simultaneously at baseline, instant after the model established, 60 min after continuous infusion of $0.01 \mu \mathrm{g} \cdot \mathrm{kg}^{-1} \cdot \mathrm{min}^{-1} \mathrm{rhBNP}$ and the time point of LVEDP $<12 \mathrm{~mm} \mathrm{Hg}$. The blood flow of the coronary were measured at rest and maximal hyperaemia. Renal angiography was performed by $4 \mathrm{~F}$ catheter and quantitative measurement of diameter was recorded by the computer assisting system. The average peak rate of renal artery $\left(\mathrm{APV}_{\mathrm{ra}}\right)$ was recorded, determination of quantitative angiography of renal artery diameter, renal vascular resistance. LVEDP and LVEF was measured.

Results 1. Coronary artery diameter increased after rhBNP administration. APV and CBF were significantly increased and CR decreased after rhBNP administration. CFR was significant rebound after continuous infusion of $0.01 \mu \mathrm{g} \cdot \mathrm{kg}^{-1} \cdot \mathrm{min}^{-1} \mathrm{rhBNP}$ for $30 \mathrm{~min}$. APV and CBF significantly increased and CR significantly decreased at the stage of infusion $0.010 \mu \mathrm{g} \cdot \mathrm{kg}^{-1} \cdot \mathrm{min}^{-1} \mathrm{rhBNP}$ in $\mathrm{rhBNP}$ Group. 2. Renal artery pressure was significantly lower after rhBNP administration. RhBNP exerts renal vasodilator effects in a dose related relationship. RBF increased gradually after administration of rhBNP and was significantly higher than control group. RVR decreased after administration of rhBNP. LVEF was lower than baseline after the models established and tended to increase after administration of rhBNP.

Conclusion It could increase blood flow of injury coronary artery, improve CFR and improve the coronary and renal haemodynamics after intravenous administration of rhBNP in pigs with AMI-ADHF.

\section{e0657 THE PERIOPERATION EFFECTS OF RECOMBINANT HUMAN B-TYPE NATRIURETIC PEPTIDE FOR HEART FAILURE PATIENTS WITH PRIMARY PERCUTANEOUS CORONARY INTERVENTION}

doi:10.1136/hrt.2010.208967.657

Fu Xianghua, Zhang Jing, Wang Xuechao, Wang Yanbo, Hao Guozhen, Fan Weize, Jiang Yunfa. The 2nd Hospital of Hebei Medical University, Shijiazhuang, Hebei, China

Objective To study the efficacy and safety of recombinant human Btype natriuretic peptide (rhBNP) in AMI-ADHF patients undergoing $\mathrm{PCI}$, especially changes in renal function and the impact of shortterm outcome during BNP treatment.

Methods 87 consecutive patients with AMI-ADHF entrolled in the study. All patients were randomly assigned to the rhBNP group and control group. rhBNP was given at $1.5 \mu \mathrm{g} \cdot \mathrm{kg}^{-1}$ intravenously and then infused intravenously $\left(0.0075-0.030 \mu \mathrm{g} \cdot \mathrm{kg}^{-1} \cdot \mathrm{min}^{-1}\right) .0 .9 \%$ Saline was used intravenously in control group as control. Clinical symptoms and killip grade were recorded. Plasma BNP levels were meaured before and after stopping the drug $6 \mathrm{~h}, 14$ days, 30 days. LVEDD and LVEF was measured. Serum creatinine (Scr) was measured before and after administered the medication $24 \mathrm{~h}, 48 \mathrm{~h}$, $72 \mathrm{~h}, 7$ days and 14 days using simplified MDRD equation to calculate estimated glomerular filtration rate. Recording the major adverse cardiac events occurrence within 30 days.

Results rhBNP group has a less dyspnoea time than the control group; The plasma BNP levels significantly lower than before treatment at different time point in the two groups. The LVEF was significantly higher in treatment group compared with baseline levels after treatment $24 \mathrm{~h}$, while LVEDD significantly decreased even after discontinuation the treatments, which remain so when the 30 days. The LVEF and LVEDD improvements in rhBNP group were significantly better than in the control group after treatment 24 h, 14 days. At day 7 after PCI, the SCr had lowered to the baseline level in the rhBNP group. The estimated glomerular filtration rate after PCI was higher in the rhBNP group than that in the control group. The occurrence of CIN was significantly lower in the rhBNP group than in the control group. The MACE event of $30 \mathrm{~d}$ in rhBNP group was significantly lower than the control group.

Conclusion rhBNP can promptly and effectively improve the heart function, reduce the incidence of major adverse cardiac events rate in acute myocardial infarction with heart failure patients, which also had a renal function protective effect in patients with and decreased incidence on CIN.

\section{e0658 ESTABLISHMENT OF YORK PIG MODEL OF ACUTE MYOCARDIAL INFARCTION WITH ACUTE DECOMPENSATED HEART FAILURE BY CORONARY OCCLUSION WITH BALLOON AND INJECTING OF MICROEMBOLUS}

doi:10.1136/hrt.2010.208967.658

Fu Xianghua, Zhang Jing, Wang Xuechao, Wang Yanbo, Miao Oing, Jiang Yunfa, Hao Guozhen, Gu Xinshun. The 2nd Hospital of Hebei Medical University, Shijiazhuang, Hebei, China

Objective To evaluate the method of yorkpig model of AMI-ADHF by coronary occlusion with balloon and injecting of microembolus 